\title{
Comparison of strength training, aerobic training, and additional physical therapy as supplementary treatments for Parkinson's disease: pilot study
}

\author{
Alessandro Carvalho $0^{1,2}$ \\ Dannyel Barbirato' \\ Narahyana Araujo' \\ Jose Vicente Martins ${ }^{3}$ \\ Jose Luiz Sá Cavalcanti ${ }^{3}$ \\ Tony Meireles Santos ${ }^{4}$ \\ Evandro S Coutinho ${ }^{5}$ \\ Jerson Laks ${ }^{1,2}$ \\ Andrea C Deslandes' \\ 'Centro de Doença de Alzheimer \\ e Outros Transtornos da Velhice, \\ Instituto de Psiquiatria, Universidade \\ Federal do Rio de Janeiro, Rio de \\ Janeiro, Brazil; ${ }^{2}$ Centro de Estudo \\ e Pesquisa do Envelhecimento, \\ Instituto Vital Brazil, Rio de Janeiro, \\ Brazil; ${ }^{3}$ Instituto de Neurologia \\ Deolindo Couto, Universidade Federal \\ do Rio de Janeiro, Rio de Janeiro, \\ Brazil; ${ }^{4}$ Departamento de Educação \\ Física da Universidade Federal de \\ Pernambuco, Pernambuco, Brazil; \\ ${ }^{5}$ Departamento de Epidemiologia \\ e Métodos Quantitativos em Saúde. \\ Escola Nacional de Saúde Pública- \\ FIOCRUZ, Rio de Janeiro, Brazil
}

Correspondence: Alessandro Carvalho Rua Ministro Gabriel de Piza 337/04/40 I Pechincha, 22770-360 Rio de Janeiro, Brazil

Email aocarvalho@gmail.com
This article was published in the following Dove Press journal:

Clinical Interventions in Aging

7 January 2015

Number of times this article has been viewed

Introduction: Physical rehabilitation is commonly used in patients with Parkinson's disease (PD) to improve their health and alleviate the symptoms.

Objective: We compared the effects of three programs, strength training (ST), aerobic training (AT), and physiotherapy, on motor symptoms, functional capacity, and electroencephalographic (EEG) activity in PD patients.

Methods: Twenty-two patients were recruited and randomized into three groups: AT (70\% of maximum heart rate), ST ( $80 \%$ of one repetition maximum), and physiotherapy (in groups). Subjects participated in their respective interventions twice a week for 12 weeks. The assessments included measures of disease symptoms (Unified Parkinson's Disease Rating Scale [UPDRS]), functional capacity (Senior Fitness Test), and EEG before and after 12 weeks of intervention. Results: The PD motor symptoms (UPDRS-III) in the group of patients who performed ST and AT improved by $27.5 \%$ (effect size $[\mathrm{ES}]=1.25$, confidence interval $[\mathrm{CI}]=-0.11,2.25$ ) and $35 \%(\mathrm{ES}=1.34, \mathrm{CI}=-0.16,2.58)$, respectively, in contrast to the physiotherapy group, which showed a $2.9 \%$ improvement $(\mathrm{ES}=0.07, \mathrm{CI}=-0.85,0.99)$. Furthermore, the functional capacity of all three groups improved after the intervention. The mean frequency of the EEG analysis mainly showed the effect of the interventions on the groups $(F=11.50, P=0.0001)$.

Conclusion: ST and AT in patients with PD are associated with improved outcomes in disease symptoms and functional capacity.

Keywords: Parkinson's disease, physical exercise, physical therapy, quality of life, functional capacity

\section{Introduction}

Parkinson's disease (PD) is currently considered the second most common neurodegenerative disorder, losing first place to Alzheimer's disease by a small margin., ${ }^{1,2}$ Functional disability caused by PD has a major impact on the lives of patients and their families. The progression of PD leads to an increasing inability to perform daily activities, loss of independence, and a decreased quality of life, and it generates socioeconomic and occupational impairments. ${ }^{3}$ Because of its complex and multifactorial etiology, which combines, in many cases, genetic, environmental, and lifestyle factors, PD is usually treated with pharmacological and surgical approaches, but these treatments are not always completely effective. ${ }^{4}$

The motor impairment in PD patients caused by bradykinesia, rigidity, tremor, and postural instability accelerates the decline in functional capacity, especially when associated with decreased activity and with a sedentary lifestyle. ${ }^{5,6}$ Physical rehabilitation may be important for the maintenance and improvement of mobility, posture, and balance in PD patients. ${ }^{7}$ Different modalities of nonpharmacological treatment such as 
physiotherapy (P), walking, running, strength training (ST), functional exercises, and whole body vibration significantly reduced the risk of falls and improved motor performance, ${ }^{8,9}$ balance and gait, ${ }^{10}$ and executive functions. ${ }^{11}$ However, further discussion is required regarding the levels of physical exertion to which PD patients may be subjected. ${ }^{12}$ In this regard, $\mathrm{P}$ appears to have lower clinical effects ${ }^{13}$ when compared with aerobic training (AT) $)^{14}$ and ST. ${ }^{15}$ However, the possible differences among these interventions have not yet been investigated in a controlled study, to the best of our knowledge.

This trial aimed to compare the effects of P, AT, and ST on improvement of the symptoms of PD. The main hypothesis of this study was that an AT and/or an ST program would be superior to $\mathrm{P}$ in improving motor symptoms, based on the Unified Parkinson's Disease Rating Scale (UPDRS) subscale UPDRS-III. The secondary outcomes studied were functional capacity (Senior Fitness Test), balance, walking speed, and electroencephalographic activity (EEG) to examine possible central nervous system changes (activity of the cerebral cortex).

\section{Materials and methods}

\section{Standard protocols and patient consent}

A prospective longitudinal, randomized, controlled intervention study was conducted from September 2010 to September 2012. Patients with idiopathic PD diagnosed by neurologist experts in movement disorders were recruited from an outpatient rehabilitation department of the Federal University of Rio de Janeiro, Brasil. The inclusion criteria were age between 45 years and 80 years, a diagnosis of PD, and stage $1-3$ on the Hoehn and Yahr scale. ${ }^{16}$ The exclusion criteria comprised any disease that hindered the application of an evaluation instrument, clinical comorbidities that make it impossible to use physical effort, individuals of New York Heart Association classes III and IV, significant physical limitations, and visual or hearing impairment. The patients were followed for 12 weeks. All subjects gave informed consent and the study was approved by the Ethics Committee of the Institute of Neurology Deolindo Couto (INDC) (protocol number 008-09-CEP).

\section{Intervention}

We decided to compare the effects of three approaches, AT, ST, and P, all of which are widely used for the prevention and treatment of a number of diseases. We applied specific programs for each method to determine if and how they were able to increase the functional capacity and reduce the symptoms of PD. The programs were equivalent with respect to the time of intervention, duration, and weekly frequency. The patients were randomized by a blinded investigator and controlled for age, motor score in terms of UPDRS-III, ${ }^{17}$ and stage of the disease as defined by Hoehn and Yahr scale. ${ }^{18}$ They participated in their respective interventions twice a week for 12 weeks. All patients performed exercise sessions supervised by trained coaches. Patients were advised not to perform additional exercises.

\section{Aerobic training}

The AT program consisted of 30 minutes of treadmill walking (Jog 500, Technogym ${ }^{\circledR}$; Italy) preceded by 5 minutes of warming and 5 minutes of postexercise recovery. The training intensity corresponded to $60 \%$ of the maximum oxygen consumption $\left(\mathrm{VO}_{2 \max }\right)$ or $70 \%$ of the maximum heart rate $\left(\mathrm{HR}_{\max }\right)$ predicted by age and determined by the formula $\mathrm{HR}_{\max }=208-(0.7 \times$ age $) .{ }^{19}$ The speed and treadmill inclination were changed so that the patients maintained the same intensity of training during the 12-week intervention. The $\mathrm{VO}_{2}$ during training was obtained by the walking equation described by the American College of Sports Medicine, $\mathrm{VO}_{2}=[0.1$ (velocity) +1.8 (velocity) $($ inclination $/ 100)+3.5]$, where the velocity is given in $\mathrm{m} / \mathrm{min} .^{20}$

\section{Strength training}

The ST program consisted of exercises for large muscle groups using equipment for leg extensions, leg curls, leg presses, chest presses, and low row. All equipment was from the line Selection $\left(\right.$ Technogym $^{\circledR}$; Italy). The daily training volume was composed of two series of eight to 12 maximum repetitions for each exercise with rest intervals of 1 minute and 30 seconds between the sets. The initial training intensity was set between $70 \%$ and $80 \%$ of the value obtained in the one repetition maximum test, and the training load was constantly adjusted to maintain the maximum repetitions. The patients were instructed to perform a warm-up on each machine with minimum load before starting the training and to perform a stretching session at the end of the training.

\section{Physiotherapy}

The $\mathrm{P}$ group program consisted of calisthenics for the upper and lower limbs, stretching, and gait training in a $12 \mathrm{~m}$ hallway, led by a physiotherapist. The sessions lasted approximately 30-40 minutes and no type of overload was used in the proposed activities.

\section{Study procedures}

All patients were evaluated for the effect of dopaminergic medication during the training period. The motor symptoms 
were assessed using UPDRS-III by a single evaluator certified by the Movement Disorder Society (AC). The global cognitive status was assessed using the Mini-Mental State Examination. $^{21}$

To assess functional capacity, the following tests (from the Senior Fitness Test) were used: ${ }^{22}$ the Chair Stand Test (CST), Arm Curl Test (ACT), 2-Minute Step Test (2-MST), Chair Sit and Reach Test (CSRT), Back Scratch Test (BST), and 8-Foot Up and Go Test (8-FT). In addition, we used the 10-Meter Walk Test (10-MWT) ${ }^{23}$ and Berg Balance Scale (Berg). ${ }^{24}$ All these tests have good reliability and validity values. $^{22}$

The EEG mean frequency (MF) was recorded and analyzed following the protocol used in a previous study from our laboratory. ${ }^{25}$ The MF may also be modified by pharmacological and environmental interventions. Physical exercise increases the MF throughout the cortex; ${ }^{26}$ however, there is no evidence in relation to patients with PD.

The patients returned to the laboratory after 3 months for follow-up. The whole process of the baseline assessment was repeated by the same examiner. Current medications and any adverse effects were recorded at each visit.

\section{Statistical analysis}

The mean and standard deviation were computed for parametric variables and the $\chi^{2}$ test was used for categorical variables for descriptive analysis. To test for the normality and homoscedasticity of the deltas (post and pre), the Shapiro-Wilk test and Levene's tests were applied, respectively.

To compare the deltas (post and pre) between groups (AT, ST, and P), one-way analysis of variance (ANOVA) was performed for all physical tests (UPDRS, 8-FT, CST, 2-MST, ACT, BST, CSRT, Berg, 10-MWT). Significant differences in ANOVA were analyzed by the Bonferroni post hoc test.

Furthermore, we decided to use analysis of effect size (ES) in addition to traditional statistics, comparing the results obtained by all interventions to assess the clinical relevance of our findings. The ES analysis was performed using the formula proposed by Cohen. ${ }^{27}$ The values of the ES were classified as trivial $(0.20 \leq \mathrm{ES}<0.50)$, moderate $(0.50 \leq \mathrm{ES}<0.80)$, and large $(\mathrm{ES} \geq 0.80)$.

For analyzing the values of EEG MF, the electrodes were divided into six areas (frontal pole, frontal, central, temporal, parietal, and occipital). ${ }^{28}$ These areas are composed of electrodes Fp1, Fp2, and Fz (frontal pole); F4, F3, F7, and F8 (frontal); C3 and C4 (central); T3, T4, T5, and T6 (temporal); P3 and P4 (parietal); and O1 and O2 (occipital). The statis- tical model employed was a three-way ANOVA to obtain comparisons among moment (pre and post), the group (ST, $\mathrm{AT}$, and P), and the area (frontal pole, frontal, central, parietal, temporal, and occipital). The statistical package SPSS ${ }^{\circledR}$ version 20.0 for Windows (IBM Corporation, Armonk, NY, USA) was used for all analyses, and the accepted significance level for this study was $P \leq 0.05$.

\section{Results}

All variables investigated showed normality and homoscedasticity and therefore parametric analyzes were performed. Twenty-two patients were randomly assigned to the three groups (Figure 1). The only difference among the groups was found in the use of dopamine agonists, most used by the P group (Table 1).

\section{Symptoms of disease}

Regarding the stages of PD (Hoehn and Yahr scale), 4.5\% of the patients were in stage I, $54.5 \%$ in stage II, and $41 \%$ in stage III. There was no significant difference between the deltas of the three groups for the subscales of the UPDRS (Table 2).

The ST group showed a large ES in UPDRS-I (ES=0.93) and UPDRS-III (ES=1.25), presenting improved behavioral and motor symptoms. The AT group presented a large ES in UPDRS-II (ES=1.12) and UPDRS-III $(E S=1.34)$. There was no effect in UPDRS-I (ES=0.00) and worsening of symptoms in UPDRS-IV (ES=-1.23). The $\mathrm{P}$ group presented a trivial ES in UPDRS-III $(E S=0.07)$ and UPDRS-I $(E S=0.21)$ and worsening of symptoms in UPDRS-II $(E S=-0.13)$ and UPDRS-IV (ES=-0.51) (Figure 2).

\section{Functional capacity}

In comparing the deltas of the functional capacity tests there was a significant difference among the groups $(F=5.65$, $P=0.01)$ in their aerobic capacity assessed by the $2-M S T$. The Bonferroni post hoc test revealed that the ST and AT groups showed significant improvement after training, which was absent in the P group. There were no statistically significant differences among the groups for the analyses of the deltas of the other functional capacity tests (Table 2).

In the analysis of ES, the ST group had a large ES in the 8-FT (ES=-1.18) and CST (ES=1.81); a moderate ES in the ACT $(\mathrm{ES}=0.74), 2-\mathrm{MST}(\mathrm{ES}=0.72)$, and 10-MWT $(\mathrm{ES}=0.78)$; and a trivial $\mathrm{ES}$ in the BST $(\mathrm{ES}=0.38)$, CSRT $(\mathrm{ES}=0.28)$, and Berg $(\mathrm{ES}=0.44)$. The AT group showed a large $\mathrm{ES}$ in the 8-FT $(\mathrm{ES}=1.08)$, ACT (ES=1.16), CST $(\mathrm{ES}=0.86)$, and 10-MWT $(\mathrm{ES}=1.20)$; a moderate $\mathrm{ES}$ in the 


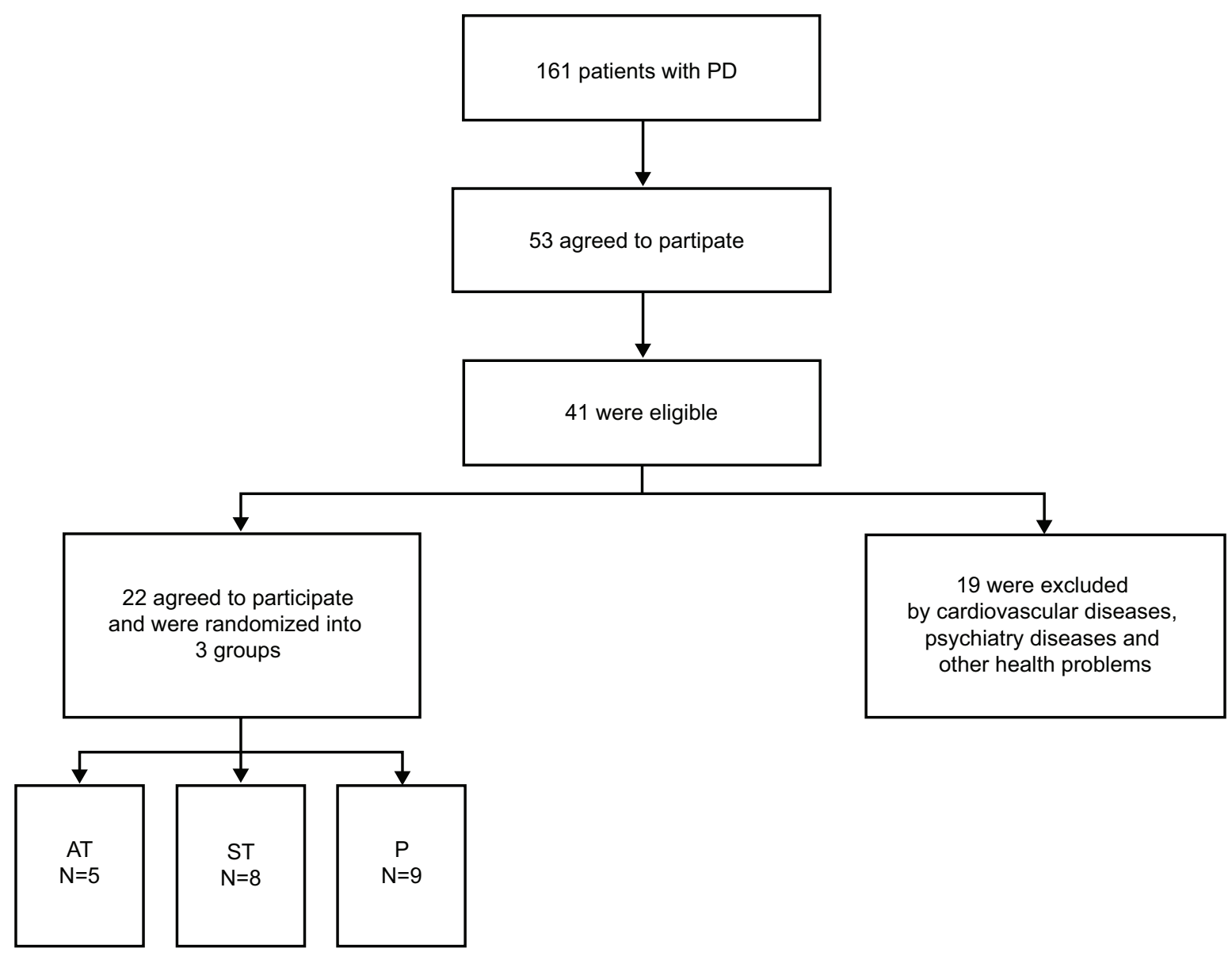

Figure I Flow diagram for identification of eligible patients.

Abbreviations: AT, aerobic training; P, physiotherapy; PD, Parkinson's disease; ST, strength training.

Table I Descriptive analysis of the three groups (physiotherapy [P], aerobic training [AT], and strength training [ST]) at baseline

\begin{tabular}{lllll}
\hline Variables & AT (n=5) mean (SD) & ST (n=8) mean (SD) & P (n=9) mean (SD) & P-value \\
\hline Education (years) & $6.8(3.2)$ & $9.8(4.8)$ & $10.7(4.5)$ & 0.312 \\
Age (years) & $64.8(11.9)$ & $64.1(9.9)$ & $62.1(11.7)$ & 0.893 \\
Sex (\% men) & 80.0 & 71.4 & 55.6 & 0.614 \\
MMSE (score) & $24.6(4.0)$ & $25.8(3.2)$ & $69.0(16.1)$ & 0.585 \\
Weight (kg) & $64.2(13.5)$ & $69.2(16.9)$ & $165.5(9.3)$ & 0.836 \\
Height (cm) & $165.4(11.6)$ & $167.5(3.4)$ & $4.3(2.8)$ & 0.877 \\
Time of disease (years) & $6.6(1.5)$ & $6.0(2.6)$ & $2.3(0.5)$ & 0.238 \\
Stage of disease (H\&Y) & $2.6(0.5)$ & $2.1(0.6)$ & $3.1(2.3)$ & 0.429 \\
UPDRS-I (score) & $3.0(2.4)$ & $3.4(1.9)$ & $10.0(4.5)$ & 0.931 \\
UPDRS-II (score) & $14.6(5.1)$ & $13.0(5.3)$ & $34.9(8.6)$ & 0.232 \\
UPDRS-III (score) & $31.0(10.0)$ & $40.4(9.7)$ & $4.4(3.0)$ & 31.8 \\
UPDRS-IV (score) & $2.8(2.0)$ & $4.2(2.8)$ & 9.1 & 0.233 \\
Levodopa (\%) & 18.2 & 36.4 & 4.5 & 0.3854 \\
Dopamine agonist (\%) & 18.2 & 27.3 & 4.5 & $0.040^{\mathrm{a}}$ \\
Amantadine (\%) & 9.1 & 18.2 & 13.6 & 0.126 \\
COMT inhibitor (\%) & 0 & 0 & & 0.487 \\
Others (\%) & 13.6 & 22.7 & & \\
\hline
\end{tabular}

Notes: Level of statistical significance $P \leq 0.05$. aSignificant difference between groups.

Abbreviations: COMT, catechol O-methyltransferase; H\&Y, Hoehn and Yahr scale; MMSE, Mini-Mental State Examination; SD, standard deviation; UPDRS, Unified Parkinson's Disease Rating Scale; UPDRS-I, behavioral; UPDRS-II, activities of daily living; UPDRS-III, motor examination; UPDRS-IV, pharmacological complications. 


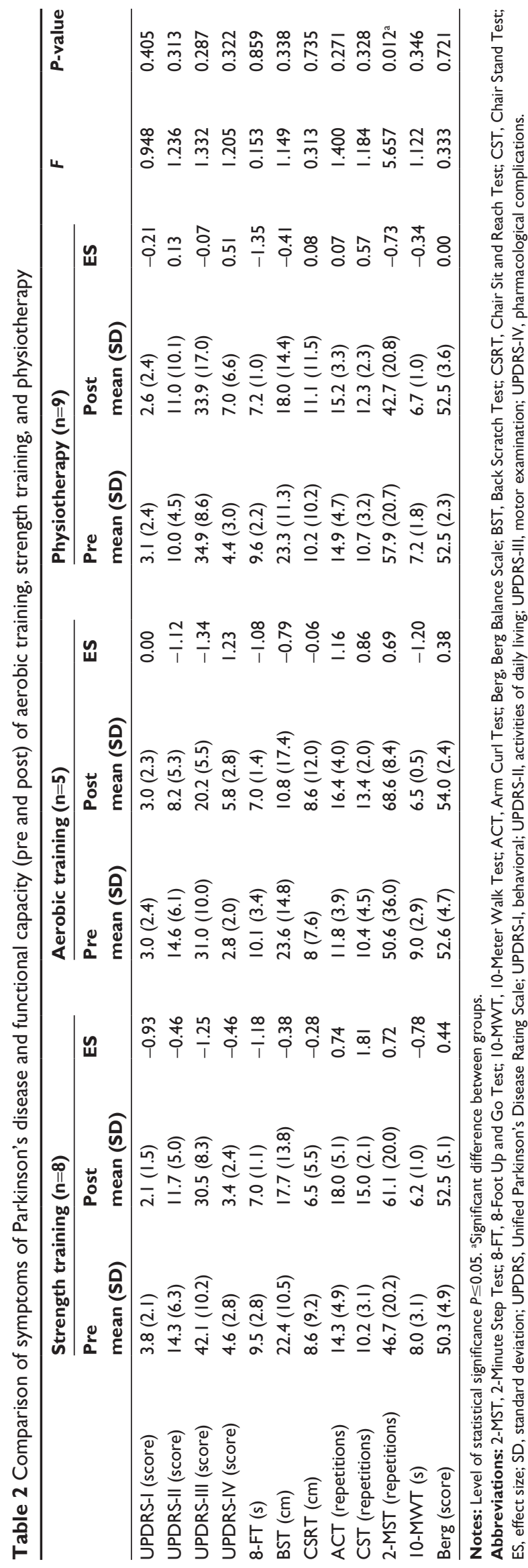

BST ( $\mathrm{ES}=0.79)$ and 2-MST ( $\mathrm{ES}=0.69)$; and a trivial $\mathrm{ES}$ in $\mathrm{Berg}(\mathrm{ES}=0.38)$. The $\mathrm{P}$ group showed a large ES in the 8-FT ( $\mathrm{ES}=1.35)$, a moderate $\mathrm{ES}$ in the $\mathrm{CST}(\mathrm{ES}=0.57)$, a trivial $\mathrm{ES}$ in the BST ( $\mathrm{ES}=0.41)$ and 10-MWT ( $\mathrm{ES}=0.34)$, and worsening of symptoms in the 2-MST (ES=-0.73) (Table 2).

\section{EEG MF}

The results of the EEG MF analysis primarily demonstrated the effect of the interventions on the groups $(F=11.50$, $P=0.00)$, without giving significance to area $(F=1.98$, $P=0.97$ ), moment $(F=0.01, P=0.89)$, and interactions between moment $\times$ group $(F=2.36, P=0.09)$, moment $\times$ area $(F=0.27$, $P=0.93)$, group $\times$ area $(F=0.21, P=0.99)$, and moment $\times$ group $\times$ area $(F=0.23, P=0.93)$. The AT and ST groups showed higher MF compared with the $\mathrm{P}$ group, which is related to increased cortical activation. Figure 3 illustrates the cortical activity of the groups using cortical maps with the delta values. The yellow and orange colors represent increased cortical activity in the anterior and posterior areas of the cortex. The violet, black, and green colors represent decreased cortical activity in the same areas.

\section{Discussion}

This study aimed to compare the effects of ST, AT, and P as supplementary treatments for the motor symptoms of PD in a randomized controlled design. Our results showed that after 3 months of intervention, the improvement in PD symptoms was similar between the groups that performed AT and ST, and this improvement was greater than that of the group that was on P sessions. To the best of our knowledge, this is the first study to compare two types of exercise (AT and ST) with a group that was submitted to $P$ as a control group. We found that the effects in the exercise groups are not only due to physical activity but possibly associated with physiological changes in response to training with a controlled overload and progression of intensity. This inference is further strengthened by the observation that the largest ES was found in the groups that had undergone AT and ST.

The groups of patients that underwent AT and ST showed significant clinical improvements in the motor symptoms of $\mathrm{PD}$, as well as in functional capacity, indicating that prescribing exercise with controlled intensity, duration, and frequency may improve the physical health of patients with PD. There was a $27.5 \%$ and $35 \%$ improvement in motor symptoms (UPDRS-III) for the ST and AT groups, respectively, in contrast to a $2.9 \%$ improvement for the P group. These results demonstrate that pharmacological treatment associated with physical exercise (AT and/or ST) may promote 


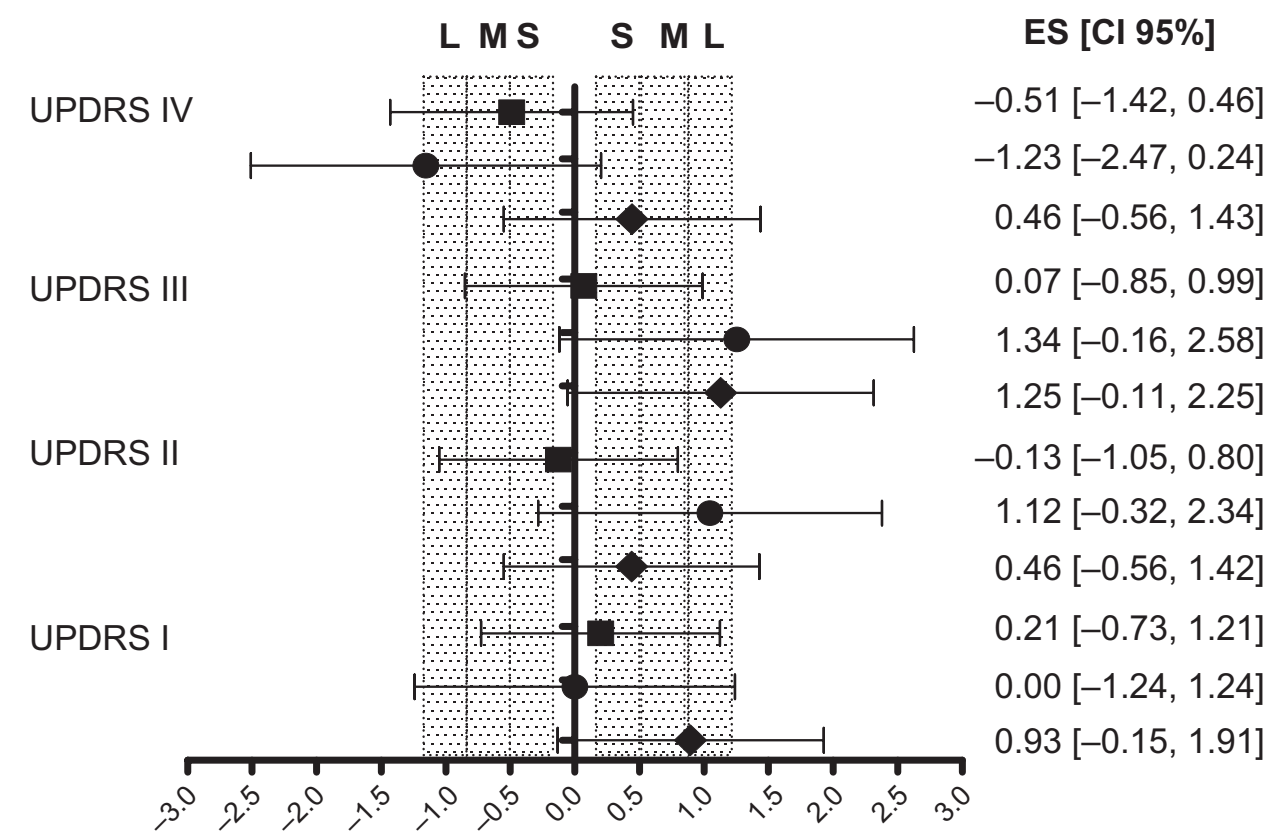

Worsening of symptoms Improvement of symptoms

Cohen's $d$ effect size

- Physiotherapy

Aerobic training

Strength training

Figure 2 Effect size (ES) of symptoms of Parkinson's disease.

Abbreviations: Cl, confidence interval; L, large; M, moderated; S, small; UPDRS, Unified Parkinson's Disease Rating Scale; UPDRS-I, behavioral; UPDRS-II, activities of daily living; UPDRS-III, motor examination; UPDRS-IV, pharmacological complications.

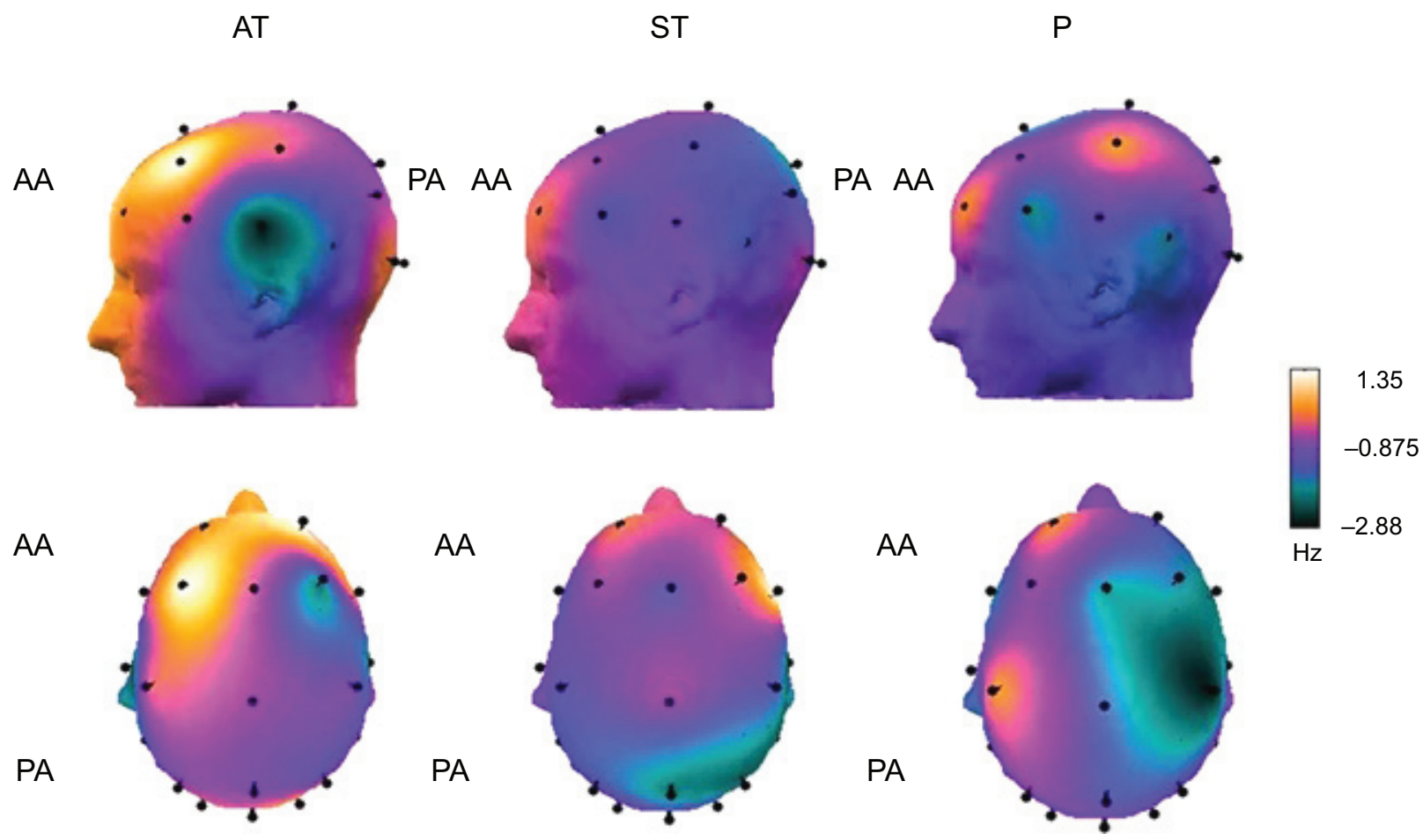

Figure 3 Spectral analysis of mean frequency by delta (post and pre) of aerobic training (AT), strength training (ST), and physiotherapy (P) groups. ${ }^{a}$ Note: ${ }^{a}$ The yellow and orange colors represent increased cortical activation after intervention.

Abbreviations: AA, anterior area; PA, posterior area. 
better results for symptoms such as rigidity and bradykinesia than just abiding to the conventional $\mathrm{P}$ usually prescribed to PD patients. Our hypothesis was also recently confirmed by Tambosco et al ${ }^{12}$ who illustrated that the inclusion of AT and ST was effective in rehabilitation programs for PD patients. Our results also corroborate the findings of Dibble et $\mathrm{al}^{29}$ Ridgel et $\mathrm{al}^{30}$ and Alberts et al ${ }^{14}$ who showed that AT and ST associated with pharmacological treatment are capable of promoting positive responses in the cardinal symptoms of PD. In a preliminary study, Dibble et al ${ }^{29}$ submitted ten PD patients for 12 weeks of an ST program with high intensity $\left(80 \% \mathrm{HR}_{\max }\right)$, lasting 45-60 minutes, three times a week. The patients showed an improvement in bradykinesia assessed by speed walking tests $(\mathrm{ES}=0.68)$ and agility $(\mathrm{ES}=0.59)$, compared with an active control group ( $\mathrm{ES}=0.07){ }^{29}$ Ridgel et $\mathrm{al}^{30}$ and Alberts et $\mathrm{al}^{14}$ observed a $35 \%$ improvement in motor symptoms after submitting PD patients for 3 weeks of forced training on a cycloergometer. ${ }^{14,30}$ In contrast to the conclusion of Alberts et al ${ }^{14}$ that forced aerobic exercise would be more effective than voluntary exercise for improving motor symptoms of PD, our results showed that there was a $35 \%$ improvement in motor symptoms on UPDRS-III in the volunteer subjects who walked on a treadmill with due control of workout intensity.

In relation to functional capacity, $\mathrm{ST}$ and $\mathrm{AT}$ significantly improved the patients' aerobic capacity, measured by the 2-MST. Moreover, by studying the ES, we found that the ST and AT groups improved in agility, strength of the upper and lower limbs, lower limb endurance, walking speed, and dynamic balance. The AT group also showed an improvement in the flexibility of the upper limbs. The group submitted to $\mathrm{P}$ also improved in agility and lower limb strength. However, the flexibility and endurance of the lower limbs worsened after the intervention period. These results demonstrate the effect of exercise on improving variables such as strength, flexibility, and especially aerobic endurance. ${ }^{12}$ There was no difference between exercise and $\mathrm{P}$ in improving variables such as agility and balance.

Concerning the EEG, the ST and AT groups showed an increase in MF compared with the P group. As far as we know, our study is also the first to use EEG results to evaluate the effect of exercise and $\mathrm{P}$ in the treatment of PD. In PD patients, diffuse slowing of EEG was present according to progression of disease, and this phenomenon implies an impairment of neural activity, with implications on cognition, executive function, and motor function. ${ }^{31,32}$ The MF is a measure comprising all frequency bands of the EEG (delta, theta, alpha, beta, and gamma) and is positively cor- related with cerebral blood flow and glucose metabolism. ${ }^{28}$ Herz et $\mathrm{l}^{33}$ observed that dopaminergic treatment appears to increase the beta and gamma frequency bands of the EEG in prefrontal and premotor areas. Our results suggest that exercise positively influences cortical activity in PD patients. During the execution of physical exercise, more cortical areas are activated, and the main hypothesis of this phenomenon is the increase in cerebral blood flow during exercise. ${ }^{28}$ Studies on other diseases such as major depression demonstrated that AT promotes a change in asymmetry ${ }^{34}$ and an increase in the MF of the EEG. ${ }^{34,35}$ Therefore, our results corroborate the evidence on the positive effect of exercise on the brain and on mental health. Likewise, Alberts et $\mathrm{al}^{14}$ demonstrated increased activity in the cortical and subcortical areas through functional magnetic resonance imaging immediately after AT.

Studies performed in animal models suggest that the physiological adaptations promoted by exercise with intensity seem to promote structural changes in the brain, mediated by increased expression of trophic factors such as brain-derived neurotrophic factor, glial cell-derived neurotrophic factor, vascular endothelial growth factor, and insulin-like growth factor 1, positively influencing brain functions (motor, $\operatorname{cog}$ nitive, and behavioral). ${ }^{14,36,37}$ Further translational studies in humans are warranted to find out whether this is also the fact in PD patients.

The present study has some limitations that should be considered. The use of a small number of subjects in each group may be associated with type II error. This limited sample size has to be taken into account when interpreting to what extent the results can be generalized, particularly given the well-known heterogeneity among PD patients. Moreover, patients with PD present several comorbidities (eg, cardiovascular and mood disorders) that were not investigated in the present study, and it is important to observe the effects of exercise in these patients. Due to this fact, we did not limit our analysis to the test of statistical significance by rejecting the null hypothesis. Instead, we looked into the magnitude of the effect, which demonstrated the clinical application of our findings. For future research, we suggest applying and controlling variables such as other training intensities, combining different approaches (combined AT and ST), and analyzing hormones and trophic factors of the patients.

\section{Conclusion}

Aerobic and strength training, controlled by parameters of intensity, duration, and frequency, showed a greater improvement than conventional physical therapy in symptoms and 
cortical activity of PD patients, confirming the need for a systematic program of training with controlled intensity. Therefore, both strength and aerobic training associated with pharmacological treatment may contribute to improving response to the treatment of PD and must be prescribed to improve the physical and mental health of patients.

\section{Acknowledgments}

This work was fully supported by Fundação de Amparo à Pesquisa do Estado do Rio de Janeiro (FAPERJ) and Coordenação de Aperfeiçoamento de Pessoal de Nível Superior (CAPES, Brasil). Special thanks go to all members of the Lanex Laboratory for academic support and mutual cooperation, in particular to friends Heitor Silveira, Thiago Guimarães, Paulo de Tarso, and José Vicente. JL and EC were supported by Conselho Nacional de Pesquisa (CNPq, Brasil), JL was supported by Fundação de Amparo à Pesquisa do Estado do Rio de Janeiro (FAPERJ) - Prioridade Rio (E-26/112.631/2012), AC was supported by Coordenação de Aperfeiçoamento de Pessoal de Nível Superior (CAPES, Brasil), and ACD was supported by FAPERJ - Jovem Cientista do Nosso Estado (E26/102.174/2013).

\section{Author contributions}

$\mathrm{AC}$ and ACD performed all measurements, conceived the original idea, developed the protocol, completed the analysis, and wrote the manuscript. DB, NA, and JVM helped with data collection, participated in protocol development, and approved the manuscript. ACD, JL, EC, JLC, JVM, and TMS provided overall supervision and revised the manuscript for intellectual content. AC performed statistical analysis and ACD supervised. All authors contributed toward data analysis and drafting and revising the paper and agree to be accountable for all aspects of the work.

\section{Disclosure}

The authors report no conflicts of interest in this work.

\section{References}

1. Schapira AH, Olanow CW. Neuroprotection in Parkinson disease: mysteries, myths, and misconceptions. JAMA. 2004;291(3):358-364.

2. Schapira AH. Neurobiology and treatment of Parkinson's disease. Trends Pharmacol Sci. 2009;30(1):41-47.

3. Morris ME, Watts JJ, Iansek R, et al. Quantifying the profile and progression of impairments, activity, participation, and quality of life in people with Parkinson disease: protocol for a prospective cohort study. $B M C$ Geriatr. 2009;9:2.

4. Connolly BS, Lang AE. Pharmacological treatment of Parkinson disease: a review. JAMA. 2014;311(16):1670-1683.

5. van Hilten JJ, Hoogland G, van der Velde EA, Middelkoop HA, Kerkhof GA, Roos RA. Diurnal effects of motor activity and fatigue in Parkinson's disease. J Neurol Neurosurg Psychiatry. 1993;56(8): 874-877.
6. van Nimwegen M, Speelman AD, Hofman-van Rossum EJ, et al. Physical inactivity in Parkinson's disease. J Neurol. 2011;258(12): 2214-2221.

7. Fox SH, Katzenschlager R, Lim SY, et al. The Movement Disorder Society evidence-based medicine review update: treatments for the motor symptoms of Parkinson's disease. Mov Disord. 2011;26 Suppl 3: S2-S41.

8. Ashburn A, Fazakarley L, Ballinger C, Pickering R, McLellan LD, Fitton C. A randomised controlled trial of a home based exercise programme to reduce the risk of falling among people with Parkinson's disease. J Neurol Neurosurg Psychiatry. 2007;78(7):678-684.

9. Caglar AT, Gurses HN, Mutluay FK, Kiziltan G. Effects of home exercises on motor performance in patients with Parkinson's disease. Clin Rehabil. 2005;19(8):870-877.

10. Ebersbach G, Edler D, Kaufhold O, Wissel J. Whole body vibration versus conventional physiotherapy to improve balance and gait in Parkinson's disease. Arch Phys Med Rehabil. 2008;89(3):399-403.

11. Tanaka K, Quadros AC Jr, Santos RF, Stella F, Gobbi LT, Gobbi S. Benefits of physical exercise on executive functions in older people with Parkinson's disease. Brain Cogn. 2009;69(2):435-441.

12. Tambosco L, Percebois-Macadre L, Rapin A, Nicomette-Bardel J, Boyer FC. Effort training in Parkinson's disease: a systematic review. Ann Phys Rehabil Med. 2014;57(2):79-104.

13. Tomlinson CL, Patel S, Meek C, et al. Physiotherapy versus placebo or no intervention in Parkinson's disease. Cochrane Database Syst Rev. 2012;8:CD002817.

14. Alberts JL, Linder SM, Penko AL, Lowe MJ, Phillips M. It is not about the bike, it is about the pedaling: forced exercise and Parkinson's disease. Exerc Sport Sci Rev. 2011;39(4):177-186.

15. Corcos DM, Robichaud JA, David FJ, et al. A two-year randomized controlled trial of progressive resistance exercise for Parkinson's disease. Mov Disord. 2013;28(9):1230-1240.

16. Hoehn MM, Yahr MD. Parkinsonism: onset, progression and mortality. Neurology. 1967;17(5):427-442.

17. Movement Disorder Society Task Force on Rating Scales for Parkinson's Disease. The Unified Parkinson's Disease Rating Scale (UPDRS): status and recommendations. Mov Disord. 2003;18(7):738-750.

18. Goetz CG, Poewe W, Rascol O, et al. Movement Disorder Society Task Force report on the Hoehn and Yahr staging scale: status and recommendations. Mov Disord. 2004;19(9):1020-1028.

19. Tanaka H, Monahan KD, Seals DR. Age-predicted maximal heart rate revisited. J Am Coll Cardiol. 2001;37(1):153-156.

20. American College of Sports Medicine. ACSM's Guidelines for Exercise Testing and Prescription. Philadelphia, PA: Lippincott Williams \& Wilkins; 2005.

21. Bertolucci PH, Brucki SM, Campacci SR, Juliano Y. [The Mini-Mental State Examination in a general population: impact of educational status]. Arq Neuropsiquiatr. 1994;52(1):1-7. Portugeuse.

22. Rikli RE, Jones CJ. Senior Fitness Test Manual. Champaign, IL: Human Kinetics; 2001.

23. Salbach NM, Mayo NE, Higgins J, Ahmed S, Finch LE, Richards CL. Responsiveness and predictability of gait speed and other disability measures in acute stroke. Arch Phys Med Rehabil. 2001;82(9): 1204-1212.

24. Scalzo PL, Nova IC, Perracini MR, et al. Validation of the Brazilian version of the Berg balance scale for patients with Parkinson's disease. Arq Neuropsiquiatr. 2009;67(3B):831-835.

25. Silveira H, Deslandes AC, de Moraes H, et al. Effects of exercise on electroencephalographic mean frequency in depressed elderly subjects. Neuropsychobiology. 2010;61(3):141-147.

26. Lardon MT, Polich J. EEG changes from long-term physical exercise. Biol Psychol. 1996;44(1):19-30.

27. Cohen J. Statistical Power Analysis for the Behavioral Sciences. 2nd Edition. Vol 2a. New York, NY: Routledge; 1988.

28. Ingvar DH, Rosen I, Johannesson G. EEG related to cerebral metabolism and blood flow. Pharmakopsychiatr Neuropsychopharmakol. 1979; 12(2):200-209. 
29. Dibble LE, Hale TF, Marcus RL, Gerber JP, LaStayo PC. High intensity eccentric resistance training decreases bradykinesia and improves quality of life in persons with Parkinson's disease: a preliminary study. Parkinsonism Relat Disord. 2009;15(10):752-757.

30. Ridgel AL, Vitek JL, Alberts JL. Forced, not voluntary, exercise improves motor function in Parkinson's disease patients. Neurorehabil Neural Repair. 2009;23(6):600-608.

31. Morita A, Kamei S, Serizawa K, Mizutani T. The relationship between slowing EEGs and the progression of Parkinson's disease. J Clin Neurophysiol. 2009;26(6):426-429.

32. Morita A, Kamei S, Mizutani T. Relationship between slowing of the EEG and cognitive impairment in Parkinson disease. J Clin Neurophysiol. 2011;28(4):384-387.

33. Herz DM, Siebner HR, Hulme OJ, Florin E, Christensen MS, Timmermann L. Levodopa reinstates connectivity from prefrontal to premotor cortex during externally paced movement in Parkinson's disease. Neuroimage. 2014;90:15-23.
34. Deslandes AC, Moraes H, Alves H, et al. Effect of aerobic training on EEG alpha asymmetry and depressive symptoms in the elderly: a 1-year follow-up study. Braz J Med Biol Res. 2010;43(6):585-592.

35. Silveira H, Deslandes A, de Moraes H, et al. Effects of exercise on electroencephalographic mean frequency in depressed elderly subjects. Neuropsychobiology. 2010;61(3):141-147.

36. Deslandes A, Moraes H, Ferreira C, et al. Exercise and mental health: many reasons to move. Neuropsychobiology. 2009;59(4):191-198.

37. Deslandes A. The biological clock keeps ticking, but exercise may turn it back. Arq Neuropsiquiatr. 2013;71(2):113-118.
Clinical Interventions in Aging

\section{Publish your work in this journal}

Clinical Interventions in Aging is an international, peer-reviewed journal focusing on evidence-based reports on the value or lack thereof of treatments intended to prevent or delay the onset of maladaptive correlates of aging in human beings. This journal is indexed on PubMed Central, MedLine,

\section{Dovepress}

CAS, Scopus and the Elsevier Bibliographic databases. The manuscript management system is completely online and includes a very quick and fair peer-review system, which is all easy to use. Visit http://www.dovepress. com/testimonials.php to read real quotes from published authors. 